\title{
Discrete dipole approximation in time domain through the Laplace transform
}

\author{
Patrick C. Chaumet, ${ }^{1}$ Ting Zhang, ${ }^{1}$ Adel Rahmani, ${ }^{2}$ Boris Gralak, ${ }^{1}$ and Kamal Belkebir ${ }^{1}$ \\ ${ }^{1}$ Aix Marseille Université, CNRS, Centrale Marseille, Institut Fresnel, UMR 7249, 13013 Marseille, France \\ ${ }^{2}$ School of Mathematical Sciences, University of Technology Sydney, Broadway NSW 2007, Australia
}

(Received 3 June 2013; published 3 December 2013)

\begin{abstract}
We present a form of the discrete dipole approximation for electromagnetic scattering computations in time domain. We show that the introduction of complex frequencies, through the Laplace transform, significantly improves the computation time. We also show that the Laplace transform and its inverse can be combined to extract the field inside a scatterer at a real resonance frequency.
\end{abstract}

DOI: 10.1103/PhysRevE.88.063303

PACS number(s): $02.60 . \mathrm{Cb}, 41.20 . \mathrm{Jb}, 42.25 . \mathrm{Fx}$

\section{INTRODUCTION}

Electromagnetic scattering by an arbitrary threedimensional structure, in time domain, is usually modelled using the finite difference in time domain (FDTD) method [1-3]. In the FDTD, one solves numerically the differential form of Maxwell's equations on a grid. A constraint of the FDTD is that the entire computational domain needs to be discretized [4]. By contrast, the discrete dipole approximation (DDA), a scattering computation method, requires that only the scatterer (or its immediate neighborhood) be discretized [5-8]. In the DDA the outgoing wave condition is automatically satisfied by using dyadic field susceptibility tensors to describe the linear response of the fields. However, in its traditional formulation, the DDA is a frequency domain method, restricted to time-harmonic fields.

Recently we generalized the DDA to handle arbitrary, nontime-harmonic electromagnetic waves. The method, detailed in Ref. [9], consists of solving the electromagnetic scattering in frequency domain, and performing a Fourier transform to generate the time evolution of electromagnetic quantities. Of course, with this approach, one drawback of the DDA is that we must solve a large system of linear equations to find the fields inside the scatterer [4]. This can be prohibitive in terms of computer memory requirements. A common way to circumvent this problem is to use an iterative method. However, such an approach requires us to calculate many times a large matrix-vector product (MVP), and to do so for all the frequencies required to accurately describe the time evolution of the fields. Therefore, the main bottleneck for the computation time is the total number of MVP required in order to achieve the desired level of convergence of the iterative method. One can decrease the number of MVP by choosing an efficient iterative method; for instance we use a combination of the generalized product-type methods based on biconjugate gradient (GPBICG) [10], a good initial value [9] and a preconditoner of Jacobi $[11,12]$, but nevertheless the convergence is still slow.

In this article we present a general strategy to reduce the number of MVPs by introducing complex frequencies into the problem via the Laplace transform. The outcome is a reduction of the number of MVPs, and hence a speedup of the computation. However, this is not the only benefit. This approach allows us to handle resonant scatterers, for instance a plasmon resonance, in time domain, something that the Fourier transform approach of Ref. [9] cannot do.
In Sec. II we briefly present the DDA method in both its time-harmonic and time domain versions, and then, in Sec. III we present the results. Finally in Sec. IV we present our conclusion.

\section{THEORY}

In this section we describe the computation of the field scattered by an arbitrary object in frequency domain (electromagnetic fields written in upper case) and in time domain (electromagnetic fields written in lower case), using the DDA.

\section{A. DDA in time-harmonic case}

As the DDA is a well known frequency domain method, we shall only describe it briefly here $[6,13]$. Consider an object with dielectric permittivity $\varepsilon$ and arbitrary shape, in free space. For each frequency $\omega$ the object is discretized into $N$ polarizable elements. Each element $j$ located at $\mathbf{r}_{j}$ has an electric polarizability $\alpha\left(\mathbf{r}_{j}, \omega\right)$, given by

$$
\begin{aligned}
& \alpha\left(\mathbf{r}_{j}, \omega\right)=\frac{\alpha_{0}\left(\mathbf{r}_{j}, \omega\right)}{1-\frac{2}{3} i k^{3} \alpha_{0}\left(\mathbf{r}_{j}, \omega\right)}, \\
& \alpha_{0}\left(\mathbf{r}_{j}, \omega\right)=\frac{3 d^{3}}{4 \pi} \frac{\varepsilon\left(\mathbf{r}_{j}, \omega\right)-1}{\varepsilon\left(\mathbf{r}_{j}, \omega\right)+2},
\end{aligned}
$$

where $d$ is the spacing of the DDA discretization lattice; $k=$ $\omega / c$ is the wave number. Then, the local fields at subunit $i$ can be written as

$$
\mathbf{E}\left(\mathbf{r}_{i}, \omega\right)=\mathbf{E}_{0}\left(\mathbf{r}_{i}, \omega\right)+\sum_{j=1, i \neq j}^{N} \mathbf{T}\left(\mathbf{r}_{i}, \mathbf{r}_{j}, \omega\right) \alpha\left(\mathbf{r}_{j}, \omega\right) \mathbf{E}\left(\mathbf{r}_{j}, \omega\right) .
$$

T denotes the dyadic field-susceptibility tensor of free space, i.e.,

$$
\begin{aligned}
\mathbf{T}\left(\mathbf{r}_{i}, \mathbf{r}_{j}, \omega\right)= & e^{(i k r)}\left[\left(3 \frac{\mathbf{r} \otimes \mathbf{r}}{r^{2}}-\mathbf{I}\right)\left(\frac{1}{r^{3}}-\frac{i k}{r^{2}}\right)\right. \\
& \left.-\left(\frac{\mathbf{r} \otimes \mathbf{r}}{r^{2}}-\mathbf{I}\right) \frac{k^{2}}{r}\right]
\end{aligned}
$$

with $\mathbf{r}=\mathbf{r}_{i}-\mathbf{r}_{j}$ and $\mathbf{I}$ the unit tensor. $\mathbf{E}_{0}\left(\mathbf{r}_{i}, \omega\right)$ is the plane wave illuminating the object such that $\left\|\mathbf{E}_{0}(\mathbf{r}, \omega)\right\|=E_{0}$. If we write Eq. (3) for all $N$ subunits forming the object, we get a linear system of size $3 N \times 3 N$ which can be written 
symbolically

$$
\mathbf{A E}=\mathbf{E}_{0},
$$

where $\mathbf{A}$ is a matrix containing the dyadic field-susceptibility tensor and the polarizabilities. Notice as the sum in Eq. (3) is performed over $i \neq j, \mathbf{A}$ is a matrix with ones on the main diagonal. The main objective of the DDA is to solve as efficiently as possible the large linear system of Eq. (5).

The linear system can be solved iteratively using a number of methods similar to the conjugate gradient method $[11,14,15]$. Irrespective of which iterative method is used (not implying that the choice of the method is unimportant; see Ref. [16]), at each step of the iterative method, one needs to compute efficiently the matrix vector product (MVP) $\mathbf{A X}$ where $\mathbf{X}$ is an estimate of the local field given by the iterative method. The iterative process is stopped when

$$
\frac{\left\|\mathbf{A} \mathbf{X}_{n}-\mathbf{E}_{0}\right\|^{2}}{\left\|\mathbf{E}_{0}\right\|^{2}}<\epsilon,
$$

where $\mathbf{X}_{n}$ is the $n$th estimate of the local field given by the iterative method, and $\epsilon$ is the desired accuracy. Once the local field is obtained at each subunit position, the scattered field can be computed as

$$
\mathbf{E}_{\mathrm{d}}(\mathbf{r}, \omega)=\sum_{j=1}^{N} \mathbf{T}\left(\mathbf{r}, \mathbf{r}_{j}, \omega\right) \alpha\left(\mathbf{r}_{j}, \omega\right) \mathbf{E}\left(\mathbf{r}_{j}, \omega\right) .
$$

To speed up the computation of the MVP at each iteration, the product is calculated via fast Fourier transform (FFT) as $\mathbf{A}$ can be written as a Toeplitz matrix [17,18]. FFT notwithstanding, the solution of Eq. (5) is still the most time consuming part of the computation. Therefore, any step toward reducing the number of MVPs in the algorithm would be a major progress for this type of computation. This is particularly true for time domain computations which requires that the corresponding time-harmonic problem be solved for several frequencies. We will get back to this problem in Sec. III.

\section{B. DDA in time domain}

In this section we consider the case where the incident field is an electromagnetic pulse with a Gaussian envelope $f(t)$ and a spectrum centered on some frequency $\omega_{0}$ :

$$
f(t)=E_{0} \exp \left[-16\left(\frac{t-t_{0}}{\tau}\right)^{2}\right] \cos \left(\omega_{0} t\right),
$$

where $\tau$ is related to the width of the envelop of the Gaussian pulse and $t_{0}$ is chosen large enough to assume that we have $f(t)=0, \forall t \leqslant 0$. We define $F(s)$, the Laplace transform of $f(t)$ as [19]

$$
F(s)=\mathscr{L}_{\beta}[f(t)]=\int_{0}^{\infty} f(t) e^{-s t} d t,
$$

with $s=\beta+i \omega$. The inverse Laplace transform is defined along a Bromwich contour as

$$
\begin{aligned}
f(t) & =\mathscr{L}_{\beta}^{-1}[F(s)]=\frac{1}{2 i \pi} \int_{\beta-i \infty}^{\beta+i \infty} F(s) e^{s t} d s \\
& =\frac{e^{\beta t}}{2 \pi} \int_{-\infty}^{\infty} F(\beta+i \omega) e^{i \omega t} d \omega .
\end{aligned}
$$

To get $\mathbf{e}_{\mathrm{d}}(\mathbf{r}, t)$ the scattered field in time domain (or the local field inside the object), one needs to compute the inverse Laplace transform of $F(s) \mathbf{E}_{\mathrm{d}}(\mathbf{r}, s)$ where $\mathbf{E}_{\mathrm{d}}(\mathbf{r}, s)$ is obtained through Eqs. (3) and (7) with a complex frequency, i.e., in the expressions of the incident plane wave, field-susceptibility tensor (FST), and polarizability, $i \omega$ is replaced with $s$. As $\beta>0$ the $e^{i \omega r / c}$ dependence of the FST becomes $e^{i \omega r / c} e^{-\beta r / c}$. In other words, the complex frequency introduces an evanescent decay in the FST.

Notice that Eq. (10) is the inverse Laplace transform which can be expressed in the form of a inverse Fourier transform, Eq. (11) [20]. At the cost of introducing manageable truncation and discretization errors, $f(t)$ can now be computed efficiently via inverse fast Fourier transform (FFT). Let $\Delta \omega$ be the discretization step in the spectral domain, for an observation time span of $T_{\max }$ and with $N_{s}$ being the number of the sample (i.e., $\Delta t=T_{\max } / N_{s}$ ), we should choose our spectral step such that $\Delta \omega \Delta t=2 \pi / N_{s}$ or equivalently $\Delta \omega=2 \pi / T_{\max }$. Notice that due to the finite bandwidth of our pulse (Gaussian envelop), we suffer no truncation error in going from Eq. (11) to a FFT. However, there remains the discretization error. The larger $\beta$, the stronger the damping in the FST, but due to the presence of the term $e^{\beta t}$ in the inverse Laplace transform, which amplifies the numerical error, parameter $\beta$ cannot exceed a maximum value $\beta_{\max }$ [21]. Finding this $\beta_{\max }$ is not easy and remains an empirical process. Wilcox $[20,21]$ proposed the following value:

$$
\beta_{\max }=2 \Delta \omega=\frac{4 \pi}{T_{\max }},
$$

and Wedepohl $[20,21]$ proposed

$$
\beta_{\max }=\frac{\ln \left(N^{2}\right)}{T_{\max }} .
$$

As we will show in Sec. III, to avoid any numerical error in the range of observation $\left[0, T_{\max }\right]$, the best value for our configuration is

$$
\beta_{\max }=\Delta \omega=\frac{2 \pi}{T_{\max }} .
$$

Notice that the expression of the polarizability in Eq. (1) introduces a set of triplets of poles given by the solutions to the following equation:

$$
\omega^{3}=-2 i \pi \frac{c^{3}}{d^{3}} \frac{\varepsilon(\omega)+2}{\varepsilon(\omega)-1} .
$$

If the scatterer has a frequency independent relative permittivity we get a single triplet of poles. If the relative permittivity is given by a single Drude-Lorentz resonance, one gets two triplets of poles, i.e., each new Drude-Lorentz resonance leads to an additional triplet of poles.

In each triplet, the poles can be deduced from each other by a $2 \pi / 3$ rotation in the complex plane. It follows that one or two poles within each triplet have a positive imaginary part. They can contribute to the Laplace transform if they are located inside the integration path, in the complex (frequencies) plane. In all the results presented in this article, it has been checked that all the poles are located outside the integration path, and therefore, the poles make no contribution to the integrals. In the case where poles are located inside the integration path, 
their contribution can be determined using two methods. The first one is based on the use of Cauchy's integral theorem, and on the evaluation of the dyadic field susceptibility at complex frequencies. The second method is to use a formulation of the DDA which uses the macroscopic field instead of the local field, thus avoiding the poles associated with the polarizability. While both formulations are strictly equivalent [13] it is usually more convenient to use the local field to compute the cross section [6] or optical forces for example [22-25].

Notice that if one is interested in the time evolution of the macroscopic field inside the object, one needs to store the electric field at all subunits for all the frequencies, hence the memory requirement would be the usual one for the DDA times the number of frequencies. On the other hand, if one only needs the scattered field at one observation point, no extra cost in memory will be incurred.

\section{RESULTS}

\section{A. Dielectric sphere with Mie resonances}

In this section we consider a homogeneous sphere of radius $a$ with relative permittivity $\varepsilon$. Notice that for this geometry we use a modified prescription for the polarizability which accounts for local-field effects, and dramatically improves the accuracy of the DDA [26,27]. The parameters of the incident pulse are $f_{0}=3.5 \mathrm{GHz}\left(\omega_{0}=2 \pi f_{0}\right.$ and $\left.\lambda_{0} \approx 85 \mathrm{~mm}\right)$ and $\tau=1.5 \mathrm{~ns}$ and the total observation time is $T_{\max }=8 \mathrm{~ns}$. We use 40 discrete frequencies across the spectrum of the pulse. The incident field is a plane wave propagating along $z$ and polarized along $x$; see Fig. 1(a). Figure 1(b) shows the incident field versus time and Fig. 1(c) shows its spectrum. The iterative method used is GPBICG with the criterion $\epsilon=10^{-6}[10,16]$. Notice that as we need to compute the local field at each subunit position for each frequency for a plane wave illumination, we can deduce from this intermediary result the scattering cross section of the sphere versus the frequency in the complex plane with

$$
C_{\text {ext }}(s)=\frac{4 \pi k}{E_{0}^{2}} \sum_{j=1}^{N} \operatorname{Im}\left[\mathbf{E}_{0}^{*}\left(\mathbf{r}_{j}, s\right) \alpha\left(\mathbf{r}_{j}, s\right) \mathbf{E}\left(\mathbf{r}_{j}, s\right)\right] .
$$

(a)
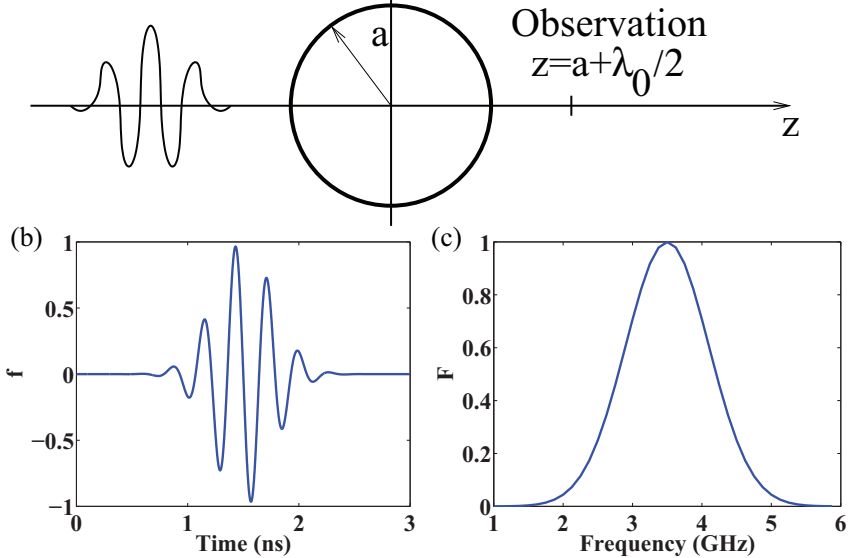

FIG. 1. (Color online) (a) Sketch of the geometry of the problem. (b) Component $x$ of the incident field vs time. (c) Spectrum of the incident field, i.e., modulus of $F(s)$ with $\beta=0$.
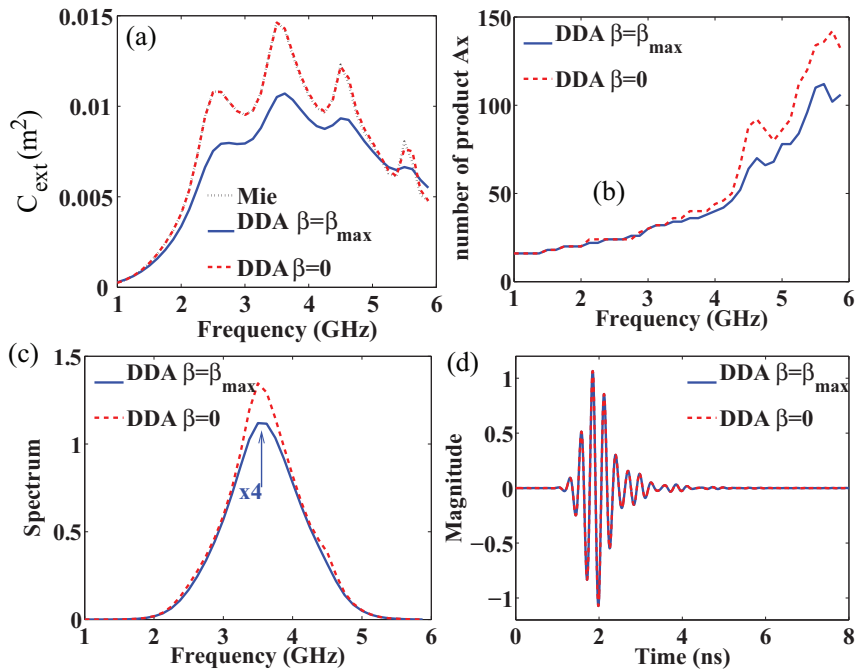

FIG. 2. (Color online) (a) Extinction cross section with Mie calculation (dotted line), DDA with $\beta=0$ (dashed line), and DDA with $\beta=\beta_{\max }$ (solid line). (b) Number of product $\mathbf{A X}$ for the iterative method to reach the convergence. (c) Spectrum of the scattered field. (d) Scattered field vs time.

Notice that when $\beta=0, C_{\text {ext }}$ is computed along the real axis which can also be evaluated with Mie theory. We also point out that the solution of Eq. (5) is faster if one uses a good initial estimate of the local field. Usually, for single frequency problems one uses the incident field, but as we are "frequency hopping," we use a linear combination of the local field obtained for the previous frequency as described in Ref. [9]. In the Appendix we present some of the details of this procedure, and give the number of MVPs for different iterative methods, for both dielectric spheres used in this section.

\section{Dielectric sphere with weak resonances}

Consider a sphere of radius $a=\lambda_{0} / 3$ with $\varepsilon=4$. Figure 2(a) shows the extinction cross section $C_{\text {ext }}$ versus frequency. The dotted line shows the prediction of Mie theory and the dashed line shows the DDA with $\beta=0$, i.e., along the real axis. Notice that the convergence of the DDA is very good as the difference between the two results is very small. The dot dashed line is computed with the value $\beta=\beta_{\max }=2 \pi / T_{\max }$. One can see that the scattering cross section computed with the damping term $\beta_{\max }$ presents weaker resonances; this is due to the fact that we compute $C_{\text {ext }}$ far from the real axis, hence far from the resonance localized on the real axis. In Fig. 2(b) the number of MVPs required by the iterative method to achieve convergence $\left(\epsilon=10^{-6}\right)$ is plotted versus frequency. When the DDA is used with $\beta=\beta_{\max }$ (solid line), the number of MVP is overall smaller than when $\beta=0$. The effect is more pronounced around the weak resonances between 4 and $5 \mathrm{GHz}$.

Figure 2(d) shows the $x$ component of the scattered field, $\mathbf{e}_{\mathrm{d}}(\mathbf{r}, t)$ estimated at $z=a+\lambda_{0} / 2$ along the $z$ axis; see Fig. 1(a). Its spectrum is given in Fig. 2(c). In time domain, we obtain the same magnitude of the field irrespective of the value of $\beta$ as demonstrated by the fact that both curves in Fig. 2(d) are superimposed. Of course, the spectra on $(\beta=0)$ and off $\left(\beta=\beta_{\max }\right)$ the real axis are different. When $\beta=\beta_{\max }$ the spectrum has the same spectral support but is damped (by 

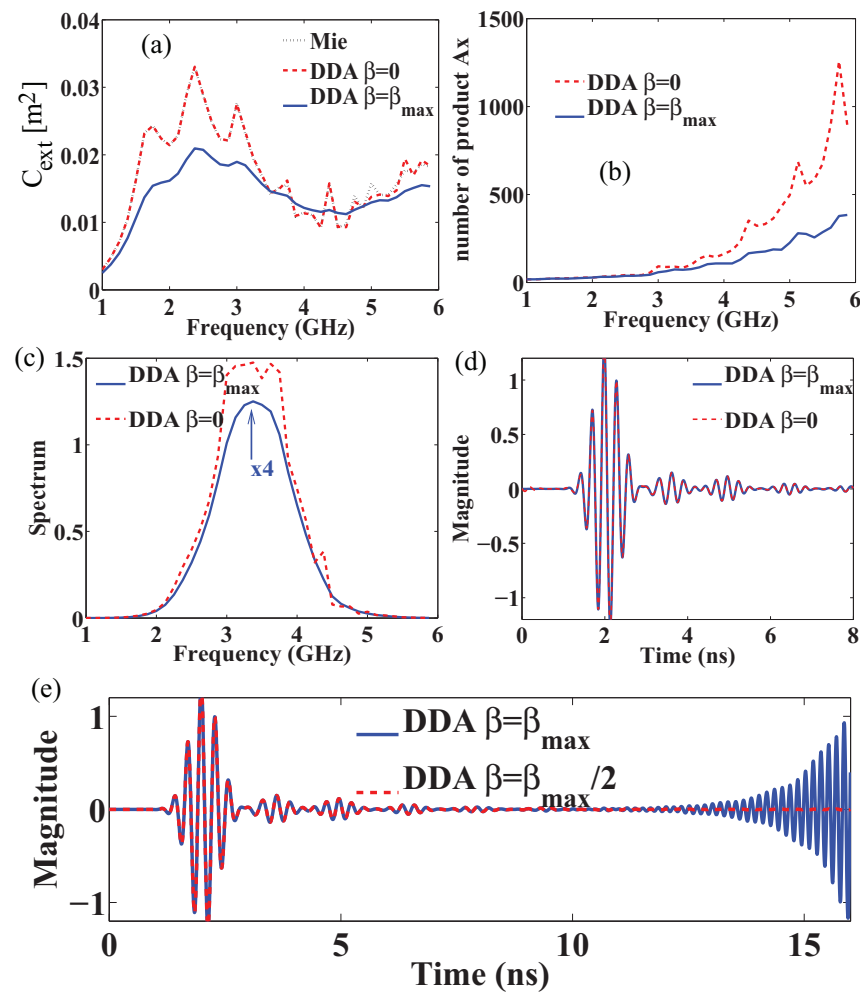

FIG. 3. (Color online) Same legend for (a)-(d) as in Figs. 2(a)2(d) respectively. (e) Scattered field vs the time for $T_{\max }^{\prime}=2 T_{\max }=$ 16 ns with $\beta=2 \pi / T_{\max }^{\prime}=\beta_{\max } / 2$ (dashed line) and $\beta=2 \pi / T_{\max }=$ $\beta_{\max }$ (solid line).

a factor 4) and smoother. This is the very reason that this new formulation of the DDA allows us to compute the fields in time domain with fewer matrix-vector products. Obviously a lower value of $\beta$ yields a less smooth spectrum and increases the number of MVPs required to achieve a given level of convergence.

Note that we checked that broadening of the frequency domain does not change $\mathbf{e}_{\mathrm{d}}(\mathbf{r}, t)$, meaning that we have no noticeable truncation error associated to the fact that a true Gaussian envelope has an unbounded support.

\section{Dielectric sphere with large Mie resonances}

We now consider a sphere with radius $a=\lambda_{0} / 2$ and $\varepsilon=4$. In Fig. 3(a) the extinction cross section $C_{\text {ext }}$ is plotted versus the frequency on the real axis and off the real axis. Compared to the example in the previous section, as the radius of the sphere has increased, the resonances on the real axis are sharper and more numerous [see the solid line in Fig. 3(a)]. In the dashed line we plot $C_{\text {ext }}$ computed with the DDA for $\beta=0$. The comparison with Mie shows that the DDA calculation has a suitable level of convergence. With $\beta=\beta_{\max }$ the extinction cross section plot is strongly smoothed as no resonance appears at high frequencies, hence we can see the strong damping effect associated with the introduction of a complex frequency. The consequence, shown in Fig. 3(b), is a strong decrease of the number of MVPs needed by the iterative method to achieve the desired level of convergence when $\beta=\beta_{\max }$. The decrease factor for the higher frequencies is about 3 compared to the case $\beta=0$ (no damping). Notice also that when $\beta=$ $\beta_{\max }$ the number of MVPs increases monotonically with the frequency, i.e., with the presence of slight resonances, contrary to $\beta=0$. This can be understood from Fig. 3(c) where the spectrum of the scattered field is plotted for both values of $\beta$. When $\beta=\beta_{\max }$ (solid line) compared to $\beta=0$ (dashed line) the spectrum is clearly smoothed and dampened by a factor 4 . In Fig. 3(d) one can see that once again, in the time domain, the computed scattered fields agree for both values of $\beta$.

If we look more closely at the time domain plot, we notice that at the end of our observation time the scattered field still exhibits small oscillations. Therefore, if we want to study the fields over a longer period of time, for instance up to when these oscillations have died out, our time interval needs to be expanded by increasing $T_{\max }$ which was $8 \mathrm{~ns}$. For example we can choose a new interval of observation $\left[0, T_{\max }^{\prime}\right]$ where $T_{\max }^{\prime}=2 T_{\max }=16 \mathrm{~ns}$. As we use an inverse FFT we need to compute twice as many frequencies with $\beta_{\max }^{\prime}=2 \pi / T_{\max }^{\prime}=$ $\beta_{\max } / 2$ [dashed line in Fig. 3(e)] lest the numerical error be magnified by the factor $e^{\beta_{\max } t}$ at large times. This means that to get a longer observation time span, one needs a dense sampling in the complex frequency domain. This is illustrated in Fig. 3(e) by the solid line. Obviously, this means that the computation time will be larger for this configuration as there are more frequencies to consider as well as, for each frequency, more steps in the iterative solution of Eq. (5) as $\beta$ has a lower value. Notice that the numerical error with $\beta_{\max }$ (solid line) becomes noticeable around $t=10 \mathrm{~ns}$ hence just after $T_{\max }=8 \mathrm{~ns}$. We have tested that the values used by Widepohl and Wilcox are too high and would introduce significant errors on the time evolution of the fields for times earlier than $T_{\max }$.

We emphasize that if the point of observation (where the fields are computed) is at a distance $r$ from the scatterer, one does not need to increase the observation time window, but should merely perform the inverse Laplace transform with the function $e^{s r / c} F(s)$ instead of $F(s)$ to perform a translation in time and keep the same time window.

\section{B. Sphere with a plasmon resonance}

In this section we consider a particle supporting a plasmon resonance. We first present a model for the illumination configuration in Fig. 1. Then we study a more realistic configuration with a gold particle illuminated by a pulse whose spectrum lies in the visible range and contains the plasmon resonance.

\section{Sphere with a weakly damped plasmon resonance}

Consider a sphere smaller than the wavelength of illumination, with a relative permittivity given by a Drude model:

$$
\varepsilon(\omega)=1-\frac{\omega_{p}^{2}}{\omega^{2}+i \omega \Gamma},
$$

where $\omega_{p}$ denotes the plasmon frequency and $\Gamma$ the damping. The sphere has a radius $a=\lambda_{0} / 10$ and supports a plasmon resonance at frequency $\omega_{p}=\sqrt{3} \omega_{0}$. Figure 4 shows the results obtained with $\Gamma=0.1 \omega_{p}$ (left column) and $\Gamma=0.03 \omega_{p}$ (right column). With strong damping, i.e., $\Gamma=0.1 \omega_{p}$, Figs. 4(a)$4(\mathrm{c})$, at the resonance the ratio of the number of MVP for $\beta=0$ 

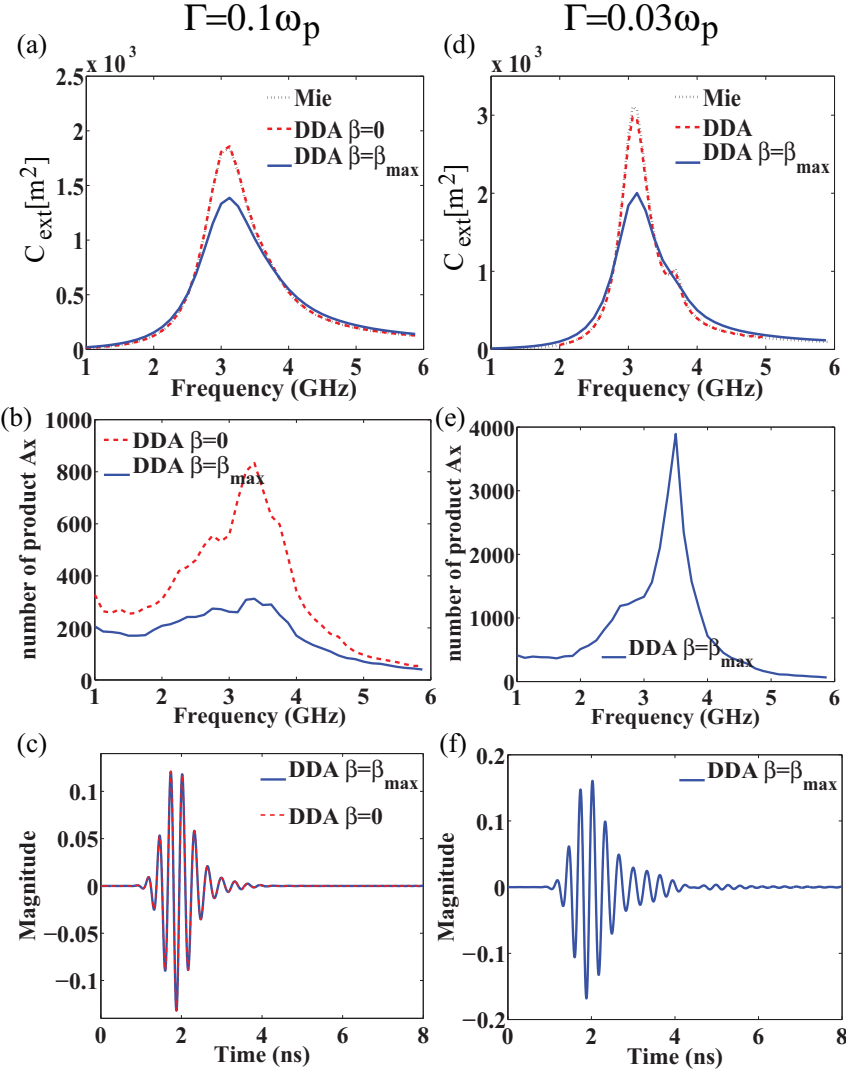

FIG. 4. (Color online) (a)-(c) $\Gamma=0.1 \omega_{p}$ : (a) Extinction cross section with Mie calculation (dotted line), DDA with $\beta=0$ (dashed line), and DDA with $\beta=\beta_{\max }$ (solid line). (b) Number of MVPs for the iterative method to reach the convergence. (c) Scattered field vs time. (d)-(f) $\Gamma=0.03 \omega_{p}$ : (d) Extinction cross section with Mie calculation (dotted line), DDA with $\beta=\beta_{\max }$ (solid line). The dashed line represents the extinction cross section obtained from the local field computed with Eq. (18). (e) Number of MVPs for the iterative method to reach the convergence. (f) Scattered field vs the time.

and $\beta=\beta_{\max }$ is at least 3. When $\Gamma=0.03 \omega_{p}$, Figs. 4(d)$4(\mathrm{f})$, the resonance is particularly sharp (dotted line) and it is strongly dampened when $\beta=\beta_{\max }$ (solid line). Notice that the DDA with $\beta=0$ fails to converge, however with $\beta=\beta_{\max }$ the number of iterations required to achieve convergence is still reasonable. The plot of the scattered field versus time shows that, due to the large resonance, an oscillation at wavelength $\lambda_{0}$ remains after the pulse has died out.

One of the problems of the DDA in the frequency domain is the handling of resonances as the iterative methods do not converge. We can use the method developed in this article to circumvent this problem. First we compute the local field $\mathbf{E}\left(\mathbf{r}_{i}, s\right)$ with $\beta=\beta_{\max }$ at each subunit position. Then, we get $\mathbf{e}\left(\mathbf{r}_{i}, t\right)$ through the inverse Laplace transform of $F(s) \mathbf{E}\left(\mathbf{r}_{i}, s\right)$. Now with a simple Fourier transform we can get $F(\omega) \mathbf{E}\left(\mathbf{r}_{i}, \omega\right)$. Then the local field at each subunit position can be obtained with

$$
\mathbf{E}\left(\mathbf{r}_{i}, \omega\right)=\frac{1}{F(\omega)} \mathscr{L}_{\beta=0}\left\{\mathscr{L}_{\beta=\beta_{\max }}^{-1}\left[F(s) \mathbf{E}\left(\mathbf{r}_{i}, s\right)\right]\right\} .
$$

Obviously when $F(\omega)$ is small, i.e., the frequency is far from the central frequency of the spectrum, the numerical error is large. However, this a not really a problem because, in that case, we are far from the resonance and the conventional DDA method works well. In Fig. 4(d), dashed line, we plot the extinction cross section calculated from the local field obtained with Eq. (18). Note that the curve is only plotted in the range $[2,5] \mathrm{GHz}$ to avoid the numerical errors associated with small values of $F(\omega)$. As we can see, the extinction cross section computed using the DDA with Eq. (18) is in good agreement with Mie theory near the plasmon resonance.

\section{Gold particle}

We now consider the scattering of light by a gold particle illuminated by a pulse with $f_{0}=6.5 \times 10^{14} \mathrm{~Hz}$ and $\tau=2.5 \times$ $10^{-14}$ (i.e., in the visible range). The relative permittivity of gold is represented by a Drude model, modified to account for the two interband transitions at $\lambda \approx 470$ and $330 \mathrm{~nm}$ :

$$
\varepsilon(\omega)=\varepsilon_{\infty}-\frac{\omega_{p}^{2}}{\omega^{2}+i \Gamma \omega}+G_{1}(\omega)+G_{2}(\omega),
$$

where constants $\omega_{p}, \Gamma, \varepsilon_{\infty}$, and functions $G_{1}(\omega), G_{2}(\omega)$ are given in Ref. [28].

a. Spherical gold particle. We first study a gold sphere with radius $a=\lambda_{0} / 10$, illuminated by a plane wave. In Figs. 5(a) and 5(b) we plot the incident field versus time and its spectrum.
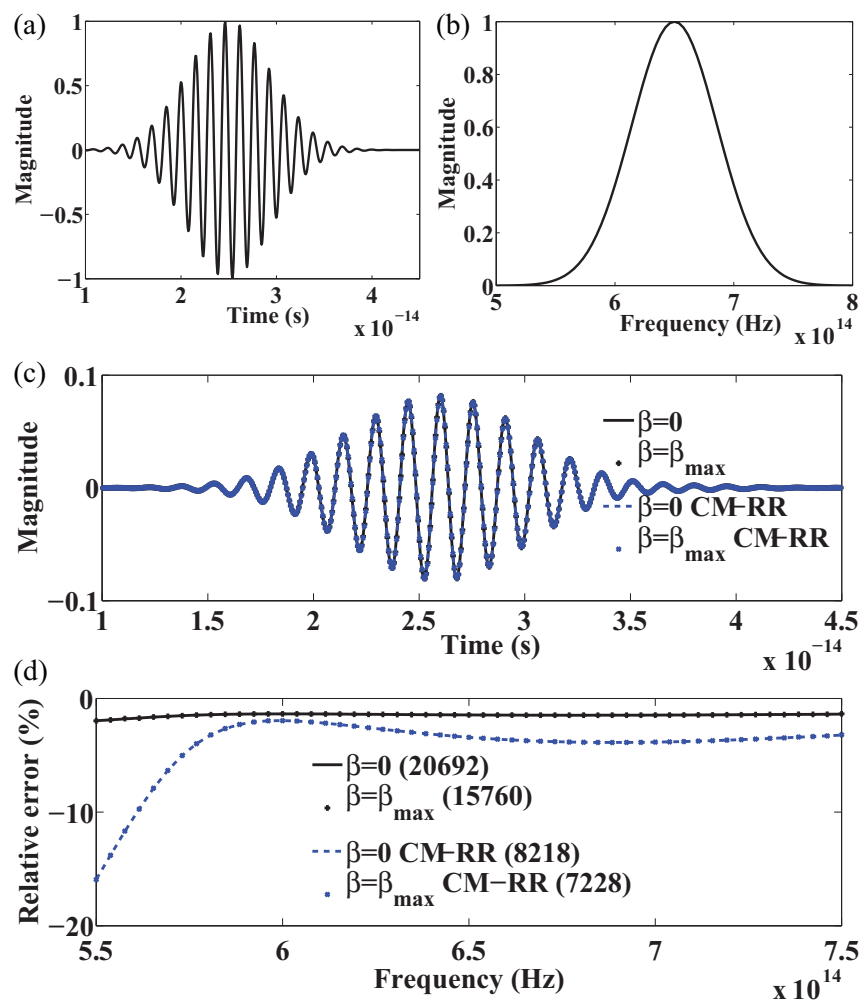

FIG. 5. (Color online) (a) $x$ component of the incident field vs time. (b) Spectrum of the incident field, i.e., modulus of $F(s)$ with $\beta=0$. (c) Scattered field at $z=a+\lambda_{0} / 2$ vs time, for $\beta=0$ and $\beta=\beta_{\max }$, for two prescriptions of the polarizability of the subunits: Clausius-Mossotti with radiative reaction term and localfield corrected formulation. (d) Relative error on the extinction cross section compared to Mie series of the Laplace transform technique with Eq. (18) for $\beta=0$ and $\beta=\beta_{\max }$ for the two polarizability prescriptions. The number of MVPs required to solve the linear system represented by Eq. (3) is given between brackets. 
Due to the strong variations of the relative permittivity of the gold in the visible range, we use a finer discretization than in the previous case, i.e., 80 discrete frequencies across the spectrum of the pulse which leads to $T_{\max }=26 \times 10^{-14} \mathrm{~s}$. Figure 5(c) shows the scattered field versus time for $\beta=0$ (solid line) and $\beta=2 \pi / T_{\max }$ (crosses). The two plots are in excellent agreement. To assess the accuracy of the Laplace method introduced in this article, we plot in Fig. 5(d) the relative error on the extinction cross section computed using Eq. (18) with $\beta=0$ (plain line) and $\beta_{m}=2 \pi / T_{\max }$ (crosses), compared to Mie theory. We see that the two curves are perfectly superimposed. Indeed, the integrand is an analytic function in the considered domain of complex frequencies, and thus its integration, between two points in the complex plane, does not depend on the path between the points. Notice that given the spherical shape of the object, as we previously indicated, we use a modified prescription for the polarizability which accounts for local-field effects [27]. To illustrate the ability of our method to compute the field in time domain using the Laplace transform irrespective of the form of the polarizability, we plot in Figs. 5(c) and 5(d) the same results (dashed line for $\beta=0$ and $\times$ for $\beta=\beta_{m}$ ) for the Clausius-Mossotti model with radiative reaction term, i.e., Eqs. (1) and (2). As we can see, the accuracy of the extinction cross section computation is slightly lowered at low frequencies due to the large value of the relative permittivity. However, in the time domain, the field computation suffers no ill effect as the weight of the low frequency components of the field is weak in the overall spectrum. In Fig. 5(d) we also see that the number of MVPs, given in brackets, increases with the modified prescription for polarizability which accounts for local-field effects.
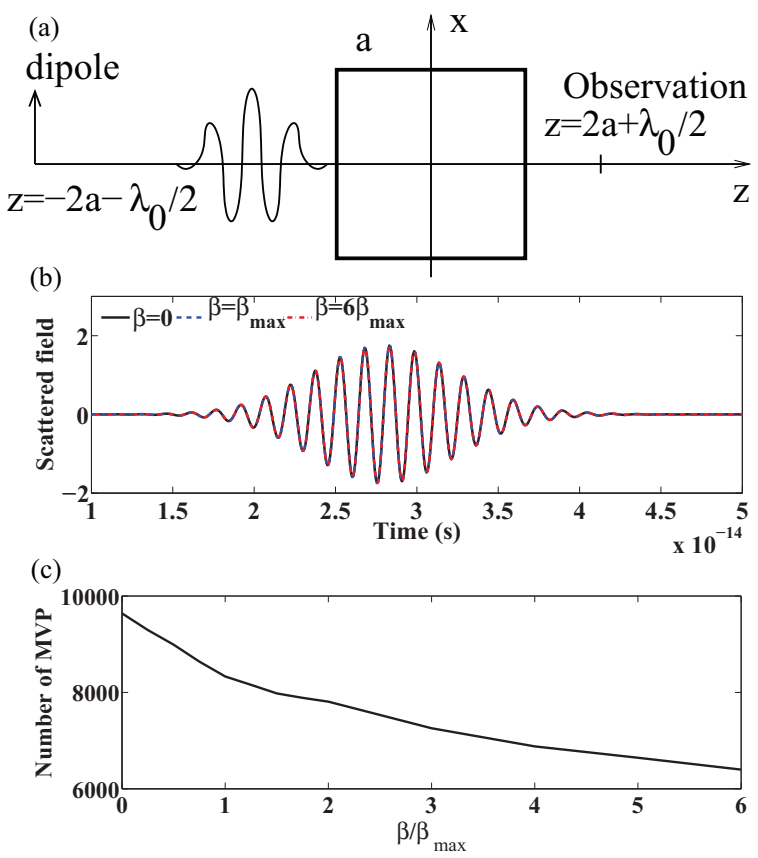

FIG. 6. (Color online) (a) Geometry of the problem. A cubic gold target is illuminated by a radiating dipole oriented along the $x$ axis. (b) Scattered field at $z=2 a+\lambda_{0} / 2$ vs time for $\beta=0$ (solid line), $\beta=\beta_{\max }$ (dashed line), and $\beta=6 \beta_{\max }$ (dot-dashed line). (c) Number of MVPs to compute all the 80 frequencies of the pulse vs the value of $\beta$. b. Cubic gold particle illuminated by a radiating dipole. In this section we study a cube with side $a=\lambda_{0} / 2$, illuminated by a dipole located at $z=-2 a-\lambda_{0} / 2$, oriented along the $x$ axis; see Fig. 6(a). We use the Clausius-Mossotti model with radiative reaction term for our polarizabilities [6]. The incident pulse is shown in Figs. 5(a) and 5(b), and is discretized using the same 80 frequencies as previously. In Fig. 6(b) we plot the scattered field at the observation point when the gold cube is illuminated by a dipole for different values of $\beta$, with $\beta_{\max }=2 \pi / T_{\max }$. As the plasmon resonance of the particle is damped by the imaginary part of material losses, we do not need to observe the fields up to time $T_{\max }$, hence the value of $\beta$ can be increased beyond $\beta_{\max }$. Since the observed scattered pulse finished before $t=5 \times 10^{-14} \approx T_{\max } / 6$, we can increase $\beta$ to $\beta=6 \beta_{\max }$. We see that all the different values of $\beta$ gives exactly the same results. In Fig. 6(c) the evolution of the number of MVPs versus the values of $\beta$ is plotted. Obviously, the larger $\beta$, the fewer MVPs.

\section{CONCLUSION}

We presented a formulation of the discrete dipole approximation (DDA) for time-domain scattering computations. We used Laplace transform techniques to efficiently solve for the fields scattered by an object, including for pathological cases, such as a scatterer exhibiting resonances (e.g., plasmon resonance, Mie resonance, etc.). Because our approach is built on the same general principles as the conventional DDA, it is not restricted to light scattering by dielectric objects. For instance, our approach can be used to compute, in the time domain, optical forces [29,30], or the scattering of an electromagnetic wave by a magnetodielectric object [31-34]. Moreover, the techniques developed in this article make it possible to compute, in the frequency regime, internal fields associated with real resonance frequency. Thus, in the time harmonic domain, our approach should be useful for the study of optical forces on resonant metal nanoparticles [35].

Note that our method can be improved by using higher value of $\beta$, however, in that case one needs to use more sophisticated Laplace transform techniques to decrease truncature and discretization error [36,37].

\section{APPENDIX: SOLUTION OF THE LINEAR SYSTEM}

In the approach introduced in this article, the crucial point is to reduce the number of MVPs required to solve iteratively the linear equation of Eq. (5). For large systems, using a good initial estimate of the fields will accelerate the convergence of the method. Accordingly, we use the solution obtained at frequency $m-1$ as the initial estimate for the computation at frequency $m$. In fact the initial estimate for the conjugate gradient method can be refined further by using several frequencies as described in Ref. [9]:

$$
\mathbf{E}_{\text {est }}\left(\omega_{m}\right)=\sum_{k=1}^{K<m} a_{k} \mathbf{E}_{\text {solution }}\left(\omega_{m-k}\right) .
$$

For the $m$ th frequency, the initial estimate is taken as a linear combination of the $K$ previous frequencies, where the 
TABLE I. Number of MVPs to achieve convergence $\left(\epsilon=10^{-6}\right)$ vs $K$, for the two different dielectric spheres studied in Sec. III A, for different values of $\beta$. The numbers correspond to the total MVPs across all frequencies.

\begin{tabular}{lccccc}
\hline \hline$K$ & 0 & 1 & 2 & 3 & 4 \\
\hline$a=\lambda / 3(\beta=0)$ & 2166 & 2064 & 2074 & 2124 & 2146 \\
$a=\lambda / 3\left(\beta=\beta_{\max }\right)$ & 1874 & 1832 & 1814 & 1814 & 1798 \\
$a=\lambda / 2(\beta=0)$ & 10538 & 9706 & 9632 & 9808 & 10002 \\
$a=\lambda / 2\left(\beta=\beta_{\max }\right)$ & 5158 & 4740 & 4700 & 4732 & 4730 \\
\hline \hline
\end{tabular}

coefficients $a_{k}$ are found by minimizing

$$
\mathcal{C}\left[\mathbf{E}_{\mathrm{est}}\left(\omega_{m}\right)\right]=\left\|\mathbf{A}\left(\omega_{m}\right) \mathbf{E}_{\mathrm{est}}\left(\omega_{m}\right)-\mathbf{E}_{0}\left(\omega_{m}\right)\right\|^{2} .
$$

The minimization procedure leads to a linear system of size $K \times K$ where the coefficients $a_{k}$ are the unknowns. In Table I we show the influence of $K$ on the number of MVPs in the frequency range used for the spheres studied in Sec. III A. The case $K=0$ corresponds to the incident field being used as the initial estimate, i.e., $\mathbf{E}_{\mathrm{est}}\left(\omega_{m}\right)=\mathbf{E}_{0}\left(\omega_{m}\right)$. We can conclude that $K=2$ or $K=3$ are reasonable choices in our case, particularly for the larger sphere.

Depending on the geometry of the scattering problem, some iterative methods may perform better than others. In Table II we present the number of MVPs for several common iterative methods, for $K=3$. We consider the conjugate gradient (CG) and a stabilized version of the biconjugate gradient (BICG) corresponding to the algorithms given in Ref. [14]. We also consider quasiminimal residual variants of the Bi-CGSTAB algorithm called QMRCGSTAB [38] and a method labeled GPBICG which is a refinement of the biconjugate gradient method [10].

For the cases considered here, GPBICG is the only iterative method to always converge, irrespective of the size of the sphere, which confirms the robustness of this method for the DDA [16]. Notice that we do not introduce any preconditioner, however, since the matrix associated with our linear system
TABLE II. Number of MVPs to achieve convergence $\left(\epsilon=10^{-6}\right)$ vs the iterative method for both spheres studied in Sec. III A, for different values of $\beta$ to compute all the frequencies. A " $x$ " means that the iterative method failed to converge. In that case the number of frequencies at which the iterative method failed is given in brackets (40 frequencies are used to describe the pulse).

\begin{tabular}{lcccc}
\hline \hline Iterative method & GPBICG & QMRCGSTAB & CG & BICG \\
\hline$a=\lambda / 3(\beta=0)$ & 2124 & 2684 & $\times(10)$ & $\times(5)$ \\
$a=\lambda / 3\left(\beta=\beta_{\max }\right)$ & 1814 & 2176 & $\times(14)$ & $\times(5)$ \\
$a=\lambda / 2(\beta=0)$ & 9808 & $\times(27)$ & $\times(8)$ & $\times(3)$ \\
$a=\lambda / 2\left(\beta=\beta_{\max }\right)$ & 4732 & $\times(39)$ & $\times(9)$ & $\times(5)$ \\
\hline \hline
\end{tabular}

has ones on the main diagonal, we have by default a Jacobi preconditioner, which is a good preconditioner for the DDA as discussed in Ref. [11]. One might decrease further the number of MVPs using a more refined preconditioner but this is outside the scope of this article.

Notice that we use a special prescription for the polarizability for a sphere which accounts for local-field effects [27]. This form of the polarizability actually increases the number of MVPs needed to satisfy the convergence criterion (taking local-field effects into account transforms the scalar polarizability into a space dependent tensor) but the resulting increased accuracy on the electric field inside the sphere is essential when dealing with plasmon or Mie resonances. For instance, in Sec. III B1 for $\Gamma=0.1 \omega_{p}\left(\Gamma=0.03 \omega_{p}\right)$ the relative error (compared to Mie) on the extinction cross section computed with DDA, using Clausius-Mossotti and radiative reaction correction, reaches more than $15 \%(150 \%)$ at low frequencies due to a large permittivity, while it is less than $5 \%(9 \%)$ with our modified polarizability. This is important because with the Laplace transform approach a large error at one frequency will affect the overall time domain computation. The same effect can be seen for the gold particle, but owing to the damping associated to a plasmon resonance, the error at low frequency can be neglected; see Figs. 5(c) and 5(d).
[1] K. S. Yee, IEEE Trans. Antennas Propagat. 14, 302 (1969).

[2] A. Taflove and M. E. Brodwin, IEEE Trans. Microwave Theor. Tech. 23, 623 (1975).

[3] A. Taflove, IEEE Trans. Antennas Propagat. 22, 191 (1975).

[4] F. M. Kahnert, J. Quant. Spectrosc. Radiat. Transfer 79-80, 775 (2003).

[5] E. M. Purcell and C. R. Pennypacker, Astrophys. J. 186, 705 (1973).

[6] B. T. Draine, Astrophys. J. 333, 848 (1988).

[7] B. T. Draine and P. J. Flatau, J. Opt. Soc. Am. A 11, 1491 (1994).

[8] M. A. Yurkin and A. G. Hoekstra, J. Quant. Spectrosc. Radiat. Transfer 106, 558 (2007).

[9] P. C. Chaumet, K. Belkebir, and A. Rahmani, Opt. Express 16, 20157 (2008).

[10] J. Tang, Y. Shen, Y. Zheng, and D. Qiu, Coastal Eng. 51, 143 (2004).

[11] P. J. Flatau, Opt. Lett. 22, 1205 (1997).

[12] J. Pestana and A. J. Wathen, J. Comput. Appl. Math. 249, 57 (2013).
[13] P. C. Chaumet, A. Sentenac, and A. Rahmani, Phys. Rev. E 70, 036606 (2004).

[14] R. D. Da Cunha and T. Hopkins, Appl. Numer. Math. 19, 33 (1995).

[15] M. A. Yurkin, V. P. Maltsev, and A. G. Hoekstra, J. Quant. Spectrosc. Radiat. Transfer 106, 546 (2007).

[16] P. C. Chaumet and A. Rahmani, Opt. Lett. 34, 917 (2009).

[17] J. J. Goodman and P. J. Flatau, Opt. Lett. 16, 1198 (1991).

[18] P. J. Flatau, G. L. Stephens, and B. T. Draine, J. Opt. Soc. Am. A 7, 593 (1990).

[19] B. Davies and B. Martin, J. Comput. Phys. 33, 1 (1979).

[20] P. Moreno and A. Ramirez, IEEE Trans. Image Process. 23, 2599 (2008).

[21] P. Gómez Zamorano and F. A. Uribe Campos, Rev. Mex. Fis. 53, 198 (2007).

[22] P. C. Chaumet and M. Nieto-Vesperinas, Phys. Rev. B 61, 14119 (2000).

[23] A. G. Hoekstra, M. Frijlink, L. B. F. M. Waters, and P. M. A. Sloot, J. Opt. Soc. Am. A 18, 1944 (2001). 
[24] P. C. Chaumet, A. Rahmani, A. Sentenac, and G. W. Bryant, Phys. Rev. E 72, 046708 (2005).

[25] P. C. Chaumet, A. Rahmani, and M. Nieto-Vesperinas, Phys. Rev. B 71, 045425 (2005).

[26] A. Rahmani, P. C. Chaumet, and G. W. Bryant, Opt. Lett. 27, 2118 (2002).

[27] A. Rahmani, P. C. Chaumet, and G. W. Bryant, Astrophys. J. 607, 873 (2004).

[28] P. G. Etchegoin, E. C. Le Ru, and M. Meyer, J. Chem. Phys. 125, 164705 (2006).

[29] P. C. Chaumet, K. Belkebir, and A. Rahmani, Phys. Rev. A 81, 021803(R) (2010).

[30] P. C. Chaumet, A. Rahmani, F. Zolla, A. Nicolet, and K. Belkebir, Phys. Rev. A 84, 033808 (2011).
[31] P. C. Chaumet and A. Rahmani, J. Quant. Spectrosc. Radiat. Transfer 110, 22 (2009).

[32] P. C. Chaumet and A. Rahmani, Opt. Express 17, 2224 (2009).

[33] P. C. Chaumet, K. Belkebir, and A. Rahmani, Opt. Express 19, 2466 (2011).

[34] M. Nieto-Vesperinas and J. J. Saenz, Opt. Lett. 35, 4078 (2010).

[35] A. S. Zelenina, R. Quidant, and M. Nieto-Vesperinas, Opt. Lett. 32, 1156 (2007).

[36] G. Honig and U. Hirdes, J. Comput. App. Math. 10, 113 (1984).

[37] A. Yonemoto, T. Hisakado, and K. Okumura, IEE Proc. Circuits Devices Syst. 150, 399 (2003).

[38] T. F. Chan, E. Gallopoulos, V. Simoncini, T. Szeto, and C. H. Tong, SIAM J. Sci. Comput. 15, 338 (1994). 